\title{
Performance Analysis of an Irreversible Regenerative Brayton Cycle Based on Ecological Optimization Criterion
}

\author{
Rajesh Kumar $^{a, *}$, S. C. Kaushik ${ }^{b}$ and Raj Kumar ${ }^{c}$ \\ ${ }^{a}$ Department of Mechanical and Automation Engineering, Amity University Haryana, Gurgaon-122413, India \\ ${ }^{b}$ Centre for Energy Studies, Indian Institute of Technology, Delhi, New Delhi-110016, India \\ ${ }^{c}$ Department of Mechanical Engineering, YMCA University of Science \& Technology, Faridabad-121006, India
}

\begin{abstract}
An ecological optimization along with a detailed parametric analysis of an irreversible regenerative Brayton cycle with finite heat capacity of external reservoirs have been carried out. The external irreversibilities due to finite temperature difference and internal irreversibilities due to fluid friction losses in compressor / turbine, regenerative heat loss, pressure loss are included in the analysis. Ecological function is thermodynamically optimized which is defined as the power output minus the product of environment temperature and entropy generation rate. A detailed analysis shows that the ecological function and corresponding power output / thermal efficiency can be maximized with judicious selection of parameters such as efficiency of turbine and compressor, effectiveness of various heat exchangers, heat source inlet temperature, pressure drop recovery coefficients and heat capacitance rate of the working fluid. It is found that the regenerative effectiveness is more prominent for maximum ecological function and corresponding thermal efficiency while cold side effectiveness is dominant factor for corresponding power output. It is also found that the effect of turbine efficiency $\left(\eta_{t}\right)$ is more than the compressor efficiency $\left(\eta_{c}\right)$ on the thermodynamic performance of an irreversible regenerative Brayton heat engine cycle. The model analyzed in this paper gives lower values of various performance parameters as expected and replicates the results of an irreversible regenerative Brayton cycle model discussed in the literature at pressure recovery coefficients of $\alpha_{1}=\alpha_{2}=1$.
\end{abstract}

Keywords: Ecological Criterion, Irreversible Brayton cycle, Regenerator, Power, Efficiency.

\section{Introduction}

Brayton cycles have been extensively used in gas power plants, aircrafts, ship propulsion and various industrial usages. Leff [3] analysed an endoreversible Brayton heat engine following Curzon and Ahlborn [1] and observed the change in Brayton cycle temperatures while altering maximum work in the cycle. Salamon and Nitzan [2] investigated the optimal operation of an endoreversible Carnot heat engine for different choices of the objective functions including maximum power, maximum efficiency, maximum effectiveness, minimum entropy production, minimum loss of availability and maximum profit. $\mathrm{Wu}$ and Kiang [4] optimized power output of a Brayton cycle

${ }^{*}$ Corresponding author. Tel.: +919467601079

E-mail: rajesharora1219@ rediffmail.com

(c) 2015 International Association for Sharing Knowledge and Sustainability

DOI: $10.5383 /$ ijtee.09.01.004 using finite time thermodynamics. Wu [5] optimized the power of an endoreversible Brayton gas heat engine. Wu \& Kiang [6] integrated real compression and expansion in Brayton heat engine and found that engine power and engine efficiency are strong functions of the compressor and turbine efficiencies. Ibrahim et al. [7] performed power optimization for a closed ideal Brayton cycle in context with various boundary configurations. Angulo-Brown [8] proposed an ecological function which is defined as power output minus the product of sink temperature and entropy generation rate. They found that the corresponding thermal efficiency of endoreversible Carnot heat engine is the average of the Carnot and Curzon-Ahlborn efficiency. Yan [9] modified ecological function [8] by replacing sink temperature with environment temperature with a justification that sink temperature is not always equal to 
environment temperature. Cheng et al. [10-11] performed ecological optimization of Brayton cycle based on endoreversible [10] and irreversible [11] configurations and observed significant decrease in entropy generation rate with a small sacrifice in power output. Kaushik et al. [12] applied finite time thermodynamic approach to an irreversible regenerative closed Brayton cycle. Tyagi et al. [13] performed ecological optimization of Stirling and Ericsson heat engines. Kaushik et al. [14] performed a thermodynamic analysis of an irreversible regenerative Brayton cycle with isothermal heat addition and optimized the power output in context with working medium temperature. They observed an improvement of $15 \%$ in the thermal efficiency of Brayton cycle with heat addition at constant temperature. Tyagi et al. [15] investigated a complex Brayton cycle under maximum ecological and found optimum values of various performance parameters at which the cycle attains maximum values of ecological function, power output and cycle efficiency.In past, many ecological optimization studies for Brayton heat engines based on endoreversible and irreversible mode have been carried out by number of researchers [16-25]. Building on this work, a further step made in this paper is to obtain expressions for maximum ecological function, power output and thermal efficiency of an irreversible regenerative Brayton cycle with pressure drop as supplementary irreversibility. The effect of effectiveness of various heat exchangers, efficiency of turbine and compressor, heat capacitance rates, heat source inlet temperature and pressure recovery coefficients have been studied in detail and the results are presented on the graphs. The model analyzed in this paper gives lower values of ecological function, power output and thermal efficiency as expected.

\section{Thermodynamic Analysis}

An irreversible regenerative Brayton cycle coupled with a heat source and heat sink of finite heat capacity is shown in Fig. 1. In this model, state 1 is the entry point of working medium at compressor and compressed up to state 2 . Then the working medium enters the regenerator where its partial heating up to state $2 \mathrm{R}$ is done by the turbine exhaust. The working medium next enters the hot side heat exchanger with a pressure drop which is reflected using pressure recovery coefficient, $\alpha_{1}=$ $\mathrm{p}_{3} / \mathrm{p}_{2}$ and heated up to state 3 , while the heat source temperature decreases from $\mathrm{T}_{\mathrm{H} 1}$ to $\mathrm{T}_{\mathrm{H} 2}$. The working medium now enters the turbine and expands up to state 4. After expansion, the working medium enters the regenerator to transfer heat partly and then enters the cold side heat exchanger with a pressure drop which is reflected using another pressure recovery coefficient, $\alpha_{2}=\mathrm{p}_{1} / \mathrm{p}_{4}$. The working medium is cooled up to state 1 , while the heat sink temperature increases from $\mathrm{T}_{\mathrm{L} 1}$ to $\mathrm{T}_{\mathrm{L} 2}$. Therefore, we consider the closed Brayton cycle 1-2-2R-3-4R-1 with real compression / expansion processes and pressure drop irreversibilities for finite heat capacity of external reservoirs. Process (1-2s) and process (3$4 \mathrm{~s})$ are isentropic in nature as shown by dotted lines in Figure 1 .

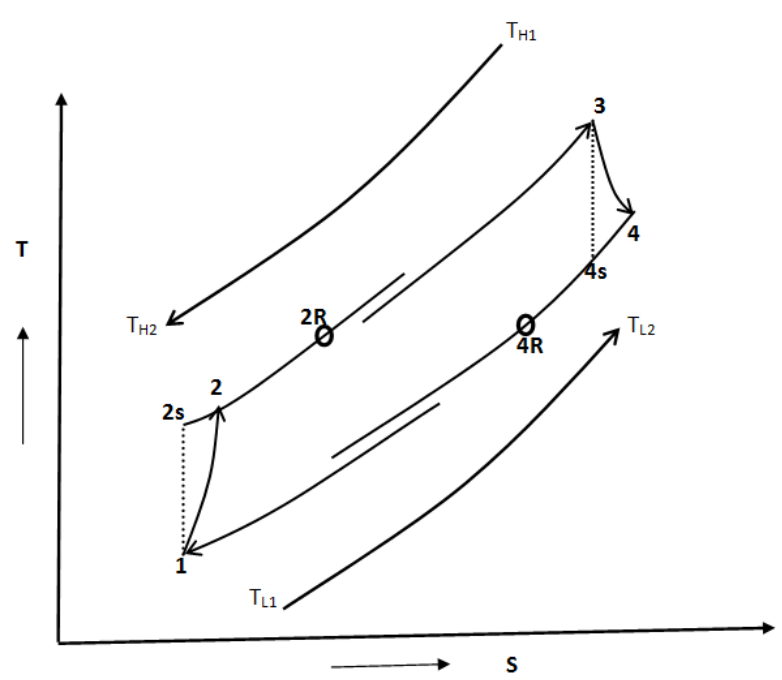

Fig 1 T-S diagram for irreversible regenerative Brayton heat engine Cycle

The hot, cold and regenerative side heat transfer rates can be presented as

$$
\begin{aligned}
& Q_{H}=U_{H} A_{H}(L M T D)_{H}=C_{H}\left(T_{H 1}-T_{H 2}\right) \\
& Q_{L}=U_{L} A_{L}(L M T D)_{L}=C_{L}\left(T_{L 2}-T_{L 1}\right) \\
& Q_{R}=U_{R} A_{R}(L M T D)_{R}=C_{W}\left(T_{4}-T_{4 R}\right)
\end{aligned}
$$

Where,

$$
\begin{aligned}
& (\text { LMTD })_{H}=\frac{\left(T_{H 1}-T_{3}\right)-\left(T_{H 2}-T_{2 R}\right)}{\ln \left\{\left(T_{H 1}-T_{3}\right) /\left(T_{H 2}-T_{2 R}\right)\right\}} \\
& (\text { LMTD })_{L}=\frac{\left(T_{4 R}-T_{L 2}\right)-\left(T_{1}-T_{L 1}\right)}{\ln \left\{\left(T_{4 R}-T_{L 2}\right) /\left(T_{1}-T_{L 1}\right)\right\}} \\
& (\text { LMTD })_{R}=\frac{\left(T_{4}-T_{2 R}\right)-\left(T_{4 R}-T_{2}\right)}{\ln \left\{\left(T_{4}-T_{2 R}\right) /\left(T_{4 R}-T_{2}\right)\right\}}
\end{aligned}
$$

From equations (1) to (6),

$$
\begin{aligned}
& Q_{H}=\varepsilon_{H} C_{H, \min }\left(T_{H 1}-T_{2 R}\right)=C_{W}\left(T_{3}-T_{2 R}\right) \\
& Q_{L}=\varepsilon_{L} C_{L, \min }\left(T_{4 R}-T_{L 1}\right)=C_{W}\left(T_{4 R}-T_{1}\right) \\
& Q_{R}=\varepsilon_{R} C_{W}\left(T_{4}-T_{2}\right)=C_{W}\left(T_{4}-T_{4 R}\right)
\end{aligned}
$$

where $\varepsilon_{\mathrm{H}}, \varepsilon_{\mathrm{L}}$ and $\varepsilon_{\mathrm{R}}$ are the effectiveness of the hot side, cold side and regenerative side heat exchangers respectively and presented as:

$$
\varepsilon_{H}=\frac{1-e^{-N_{H}\left(1-C_{H, \text { min }} / C_{H, \max }\right)}}{1-\frac{C_{H, \min }}{C_{H, \max }} e^{-N_{H}\left(1-C_{H, \min } / C_{H, \max }\right)}}
$$


$\varepsilon_{L}=\frac{1-e^{-N_{L}\left(1-C_{L, \text { min }} / C_{L, \text { max }}\right)}}{1-\frac{C_{L, \text { min }}}{C_{L, \text { max }}} e^{-N_{L}\left(1-C_{L, \text { min }} / C_{L, \text { max }}\right)}}$

$\varepsilon_{R}=\frac{N_{R}}{1+N_{R}}$

The various heat capacitance rates and number of heat transfer units can be calculated as:

$C_{H, \min }=\min \left(C_{H}, C_{W}\right) ; C_{H, \max }=\max \left(C_{H}, C_{W}\right) ;$

$C_{L, \min }=\min \left(C_{L}, C_{W}\right) ; C_{L, \max }=\max \left(C_{L}, C_{W}\right)$

and $N_{H}=\frac{U_{H} A_{H}}{C_{H, \text { min }}} ; N_{L}=\frac{U_{L} A_{L}}{C_{L, \text { min }}} ; N_{R}=\frac{U_{R} A_{R}}{C_{W}}$

The compressor and turbine efficiencies can be written as:

$\eta_{c}=\frac{T_{2 s}-T_{1}}{T_{2}-T_{1}}$

$\eta_{t}=\frac{T_{3}-T_{4}}{T_{3}-T_{4 s}}$

Now from equations (7) to (14),

$T_{4 R}=\left(1-\varepsilon_{R}\right) T_{4}+\varepsilon_{R} T_{2}$

$T_{2 R}=\left(1-\varepsilon_{R}\right) T_{2}+\varepsilon_{R} T_{4}$

$T_{1}=(1-b) T_{4 R}+b T_{L 1}$

$T_{3}=(1-a) T_{2 R}+a T_{H 1}$

$T_{2 s}=\left(1-\eta_{c}\right) T_{1}+T_{2} \eta_{c}$

$T_{4 s}=\left(1-\eta_{t}^{-1}\right) T_{3}+T_{4} \eta_{t}^{-1}$

where $a=\frac{C_{H} \varepsilon_{H}}{C_{W}} ; b=\frac{C_{L} \varepsilon_{L}}{C_{W}}$

From second law of thermodynamics for given model,

$T_{1} T_{3}=\alpha T_{2 s} T_{4 s}$

where $\alpha=\left(\alpha_{1} \alpha_{2}\right)^{\frac{k-1}{k}}$ and $\mathrm{k}$ is specific heat ratio of the working fluid.
Substituting the values of $T_{1}, T_{3}, T_{2 s}$ and $T_{4 s}$ from equations (17) - (20) into equation (21), we get the quadratic equation in $\mathrm{T}_{2}$ as:

$X T_{2}^{2}+Y T_{2}+Z=0$

Parameters $\mathrm{X}, \mathrm{Y}$ and $\mathrm{Z}$ are given in the nomenclature. Solving equation (22) for $\mathrm{T}_{2}$ gives,

$T_{2}=\frac{-Y+\sqrt{Y^{2}-4 X Z}}{2 X}$

From the first law of thermodynamics,

$P=Q_{H}-Q_{L}$

$=\varepsilon_{H} C_{H, \min }\left(T_{H 1}-T_{2 R}\right)-\varepsilon_{L} C_{L, \min }\left(T_{4 R}-T_{L 1}\right)$

Substituting the value of $\mathrm{T}_{2 \mathrm{R}}$ and $\mathrm{T}_{4 \mathrm{R}}$ from equation (15-16) into equation (24) and (7), $\mathrm{P}$ and $\mathrm{Q}_{\mathrm{H}}$ can be written as:

$P=z_{6}-x_{7} T_{2}-y_{7} T_{4}$

$Q_{H}=z_{7}-x_{8} T_{2}-y_{8} T_{4}$

Parameters $\mathrm{x}_{7}, \mathrm{x}_{8}, \mathrm{y}_{7}, \mathrm{y}_{8}, \mathrm{z}_{6}$ and $\mathrm{z}_{7}$ are given in the nomenclature.

The objective function of ecological optimization which is proposed by Angulo-Brown [8] and modified by Yan [9] is given as:

$$
E=P-T_{0} S_{g e n}
$$

Where $\mathrm{T}_{0}$ is environment temperature and $\mathrm{S}_{\text {gen }}$ is entropy generation rate. $E=P-T_{0}\left(\frac{Q_{L}}{T_{L 1}}-\frac{Q_{H}}{T_{H}}\right)$

Substitution of equations (7), (8) and (25) into equation (27),

$E=z_{9}-x_{10} T_{2}-y_{10} T_{4}$

Parameters $\mathrm{z}_{9}, \mathrm{x}_{10}$ and $\mathrm{y}_{10}$ are given in nomenclature.

Thus, optimizing equation (28) with respect to $\mathrm{T}_{4}$ i.e.

$\frac{\partial E}{\partial T_{4}}=0$ and solving for $\mathrm{T}_{4}$, gives:

$X_{1} T_{4}^{2}+Y_{1} T_{4}+Z_{1}=0$

Parameters $\mathrm{X}_{1}, \mathrm{Y}_{1}$ and $\mathrm{Z}_{1}$ are given in the nomenclature. Solving equation (29) for $T_{4}$, we get the optimum value of $T_{4}$ as

$T_{4, o p t}=\frac{-Y_{1}-\sqrt{Y_{1}^{2}-4 X_{1} Z_{1}}}{2 X_{1}}$ 


\section{Results and discussions}

In order to have numerical appreciation of the results, the effects of various performance parameters viz. efficiency of turbine and compressor, effectiveness of various heat exchangers, reservoir temperature ratio, pressure drop recovery coefficients and heat capacitance rate of the working fluid on an irreversible regenerative Brayton heat engine model are investigated. Each one of above mentioned parameter is examined by keeping rest parameters constant as $\varepsilon_{\mathrm{H}}=\varepsilon_{\mathrm{L}}=$ $\varepsilon_{\mathrm{R}}=0.75, \mathrm{~T}_{\mathrm{H} 1}=1250 \mathrm{~K}, \mathrm{~T}_{\mathrm{L} 1}=300 \mathrm{~K}, \mathrm{~T}_{0}=295 \mathrm{~K}, \eta_{\mathrm{t}}=\eta_{\mathrm{c}}=0.8$, $\mathrm{C}_{\mathrm{W}}=1.05 \mathrm{kWK}^{-1}, \mathrm{C}_{\mathrm{H}}=\mathrm{C}_{\mathrm{L}}=1 \mathrm{kWK}^{-1}, \mathrm{U}_{\mathrm{H}}=\mathrm{U}_{\mathrm{L}}=\mathrm{U}_{\mathrm{R}}=2.0 \mathrm{kWK}^{-1} \mathrm{~m}$ ${ }^{2}, \alpha_{1}=\alpha_{2}=0.95$. The obtained results are presented on graphs and discussed in detail as follows:

\subsection{Effect of $\varepsilon_{H}, \varepsilon_{L}$ and $\varepsilon_{R}$}

The variations of hot side, cold side and regenerative side effectiveness on different performance parameters are shown in figures 2(a) to 2(c). It is clearly seen from these results that maximum ecological function, power output and thermal efficiency increases as the effectiveness on either side of heat exchanger $\left(\varepsilon_{\mathrm{H}}, \varepsilon_{\mathrm{L}}\right.$ and $\left.\varepsilon_{\mathrm{R}}\right)$ is increased. It is also found that the effect of $\varepsilon_{\mathrm{L}}$ is more prominent for power output while $\varepsilon_{\mathrm{R}}$ is dominant factor for ecological function and thermal efficiency. The results obtained can also be correlated with heat transfer area. It is required to increase the heat transfer area as the effectiveness is increased which results in increase of cost of the system. So, judicious selection of effectiveness of various heat exchangers is required. However, in general, the variations of various performance parameters with respect to effectiveness are not linear and it is further seen from these results that at an effectiveness of 0.8 on either side of the heat exchanger, the better the performance of the cycle.

\subsection{Effect of heat capacitance rates $\left(C_{H}, C_{L}\right.$ and $\left.C_{W}\right)$}

The variations of heat capacitance rates of source side, sink side and cycle working fluid on maximum ecological function, power output thermal efficiency are show in figures

3 (a) to 3(c). It is clearly observed from these results that maximum ecological function, power output and thermal efficiency increases with increase in heat capacitance rates of source side and sink side reservoirs where as all the performance parameters shows steep fall with the increase in heat capacitance rate of cycle working fluid. It is also found that sink side heat capacitance rate is more dominant than source side on all the performance parameters of the cycle.

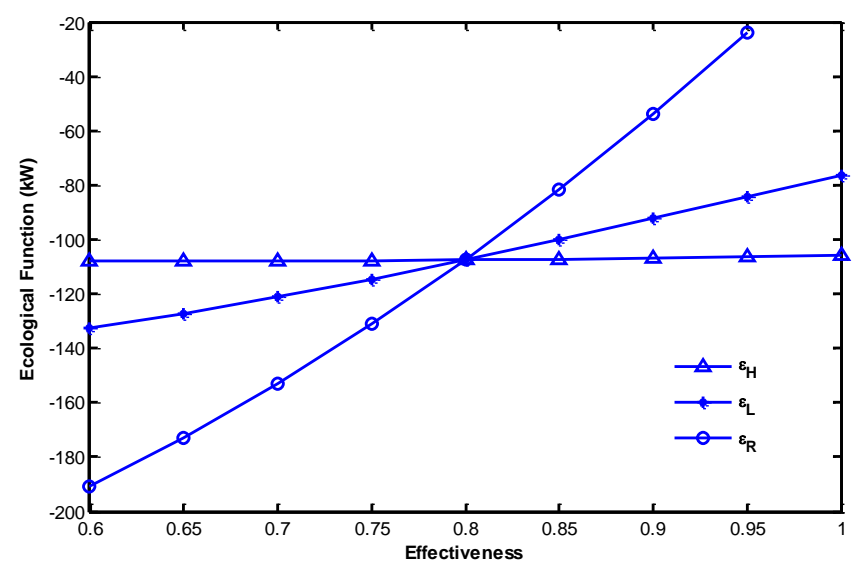

Fig 2(a) Variations of Ecological with respect to effectiveness of heat exchangers $\left(\varepsilon_{\mathrm{H}}, \varepsilon_{\mathrm{L}}\right.$ and $\left.\varepsilon_{\mathrm{R}}\right)$

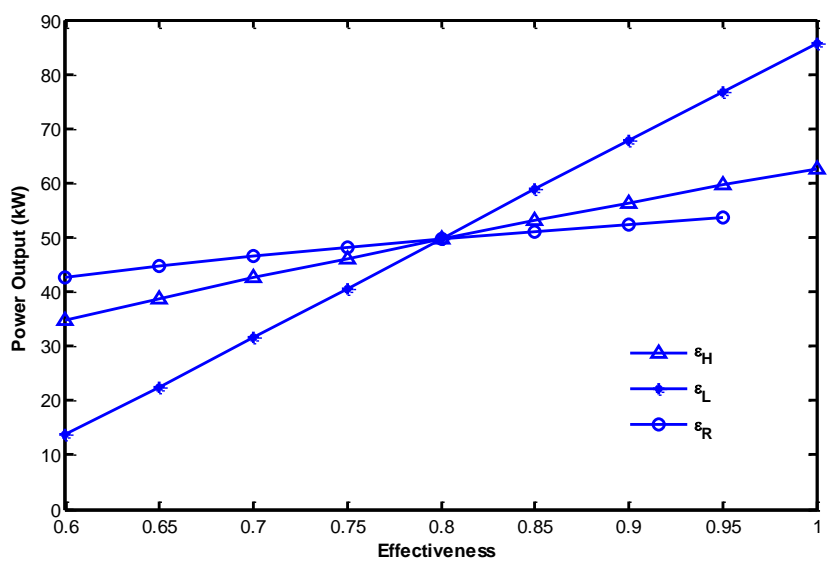

Fig 2 (b) Variations of Power Output with respect to effectiveness of heat exchangers

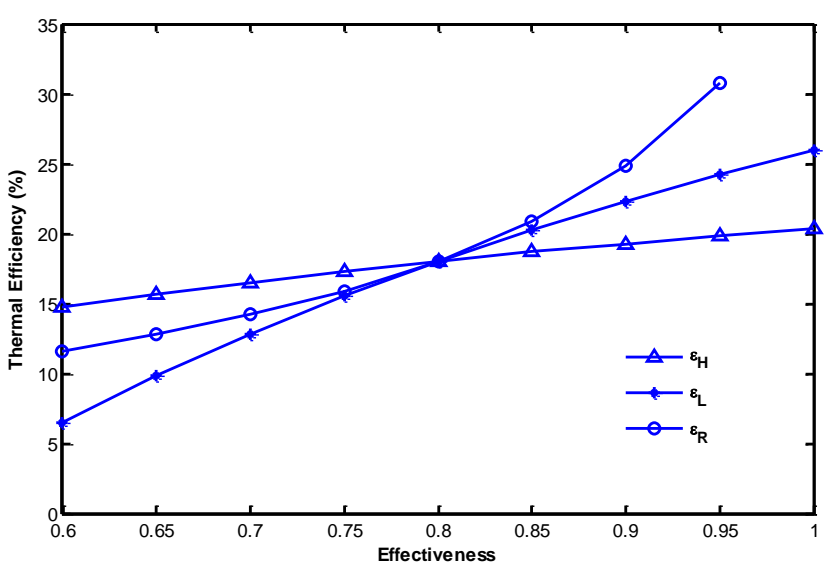

Fig 2(c) Variations of Thermal Efficiency with respect to effectiveness of heat exchangers $\left(\varepsilon_{H}, \varepsilon_{L}\right.$ and $\left.\varepsilon_{R}\right)$

\subsection{Effects of turbine and compressor efficiencies}

The variations of turbine and compressor efficiencies on maximum power output and corresponding thermal efficiency of an irreversible regenerative Brayton heat engine cycle with finite capacity heat reservoir are shown in figures 4(a) to 4(c). It is seen from these figures that maximum ecological function, power output and thermal efficiency increases with the increase in component efficiencies $\left(\eta_{t}\right.$ and $\left.\eta_{c}\right)$ which indicates that larger the component efficiency is, better the performance of the cycle. It is also found that the effect of turbine efficiency $\left(\eta_{t}\right)$ is more than the compressor efficiency $\left(\eta_{c}\right)$ on the thermodynamic performance of an irreversible regenerative Brayton heat engine cycle. Hence, for practical Brayton heat engine, lots of research and investigation is still required on compressor efficiency. 


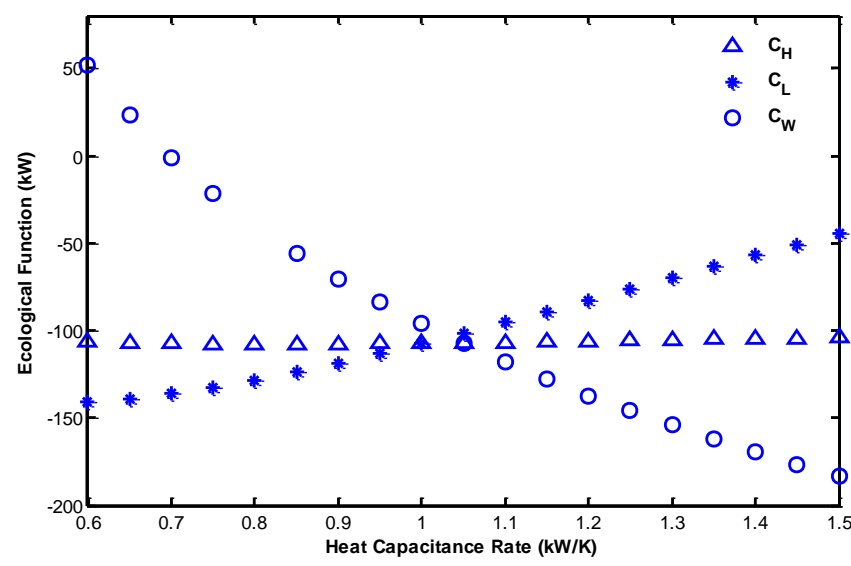

Fig 3 (a) Variations of Ecological Function with respect to heat capacitance rates $\left(\mathrm{C}_{\mathrm{H}}, \mathrm{C}_{\mathrm{L}}, \mathrm{C}_{\mathrm{W}}\right)$

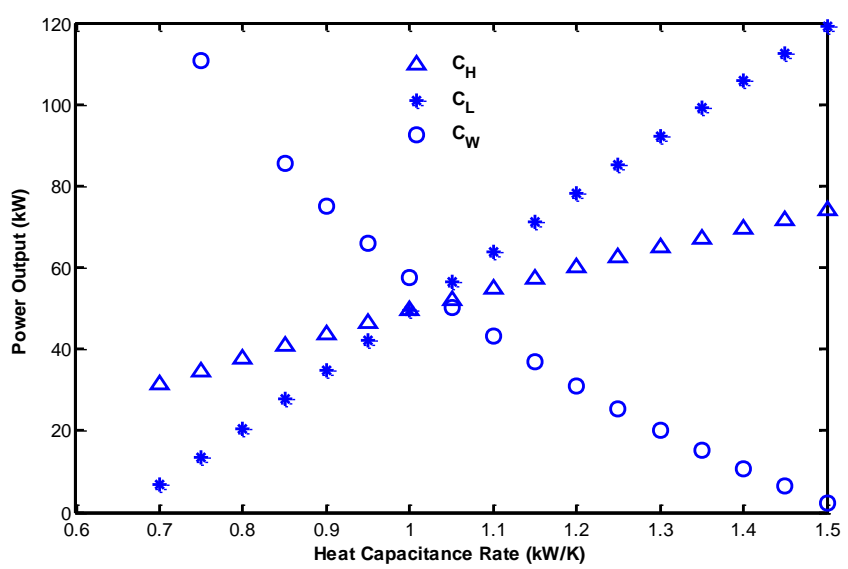

Fig 3 (b) Variations of Power Output with respect to heat capacitance rates $\left(\mathrm{C}_{\mathrm{H}}, \mathrm{C}_{\mathrm{L}}, \mathrm{C}_{\mathrm{W}}\right)$

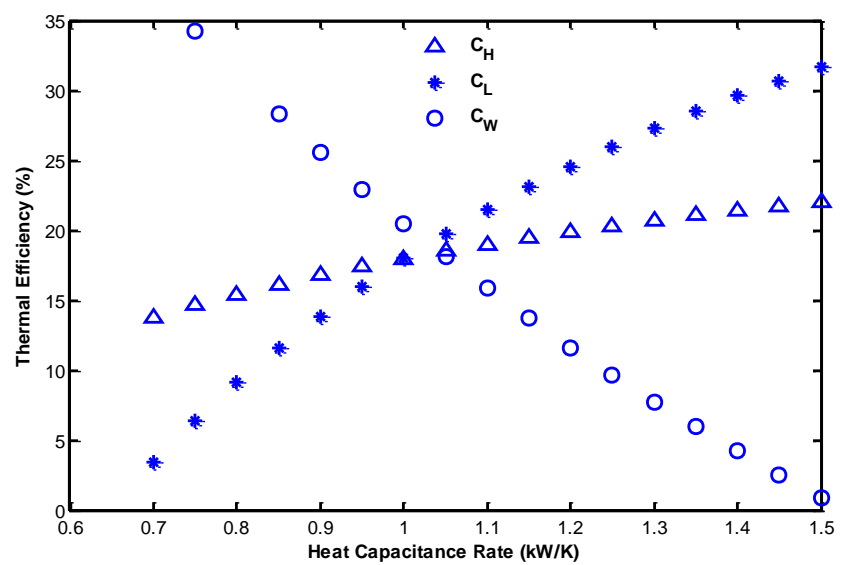

Fig 3 (c) Variations of Thermal Efficiency with respect to heat capacitance rates $\left(C_{H}, C_{L}, C_{W}\right)$

\subsection{Effects of pressure recovery coefficients}

Figure 5(a) and figure 5(b) shows the effect of pressure recovery coefficients on various performance parameters of an irreversible regenerative Brayton heat engine cycle. It is seen from these figures that maximum ecological function, power output and thermal efficiency increases as the pressure drop is decreased. It is also seen from these figures that various performance parameters attains their maximum value at zero pressure drop which cannot be achieved in realistic Brayton heat engine cycle. Further, maximum ecological function, power output and thermal efficiency reflect linear variations with pressure recovery coefficients.

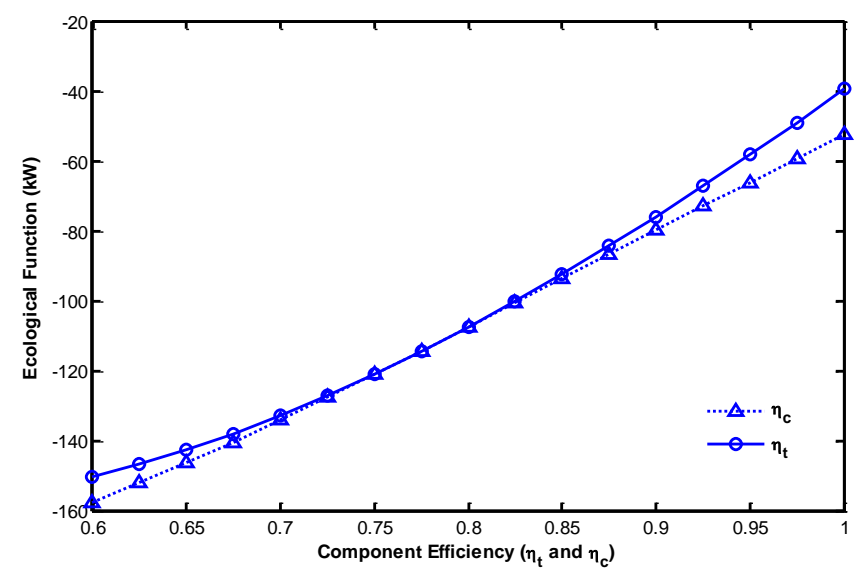

Fig 4(a) Variations of Ecological Function with respect to component efficiency $\left(\eta_{c}\right.$ and $\left.\eta_{t}\right)$

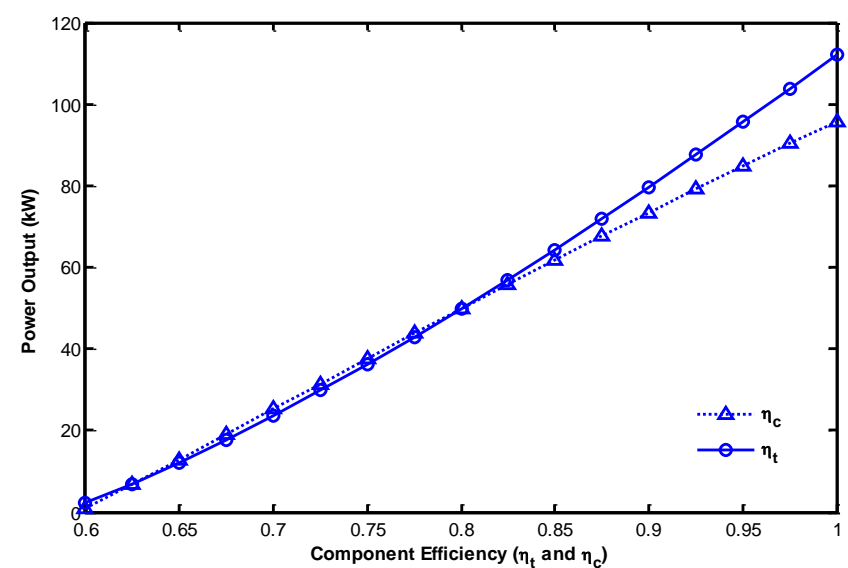

Fig 4(b) Variations of Power Output with respect to component efficiency $\left(n_{c}\right.$ and $\left.n_{t}\right)$

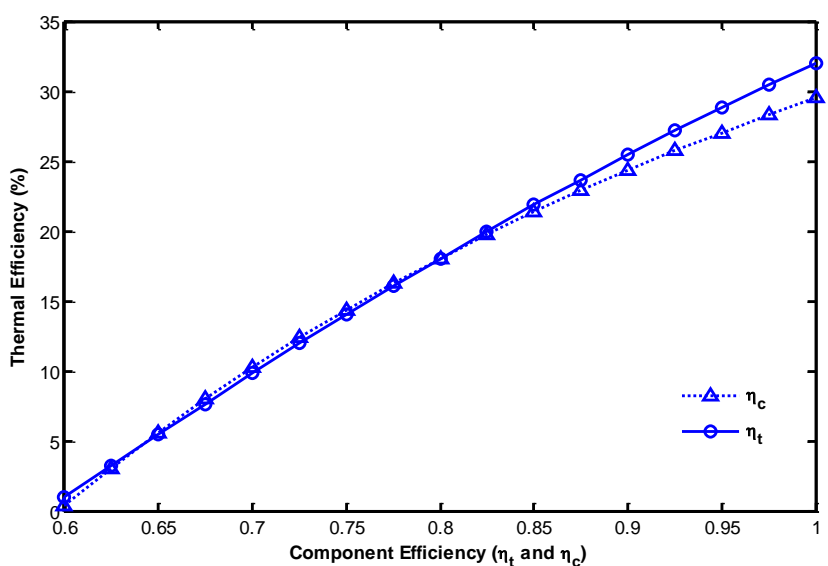

Fig 4(c) Variations of Thermal Efficiency with respect to component efficiency $\left(\eta_{c}\right.$ and $\left.\eta_{t}\right)$

3.5 Effects of reservoir temperature ratio $\left(T_{H 1} / T_{L 1}\right)$

The variation of reservoir temperature ratio on various performance parameters of an irreversible regenerative Brayton heat engine cycle are shown in figure 6(a) and 6(b). It is seen from these figures that power output and thermal efficiency increases while maximum ecological function decrease with increase in $\mathrm{T}_{\mathrm{H} 1} / \mathrm{T}_{\mathrm{L} 1}$ ratio. Further, $\mathrm{T}_{\mathrm{H} 1} / \mathrm{T}_{\mathrm{L} 1}$ ratio is increased either by decreasing sink temperature or by increasing source temperature. Again, it is not practical to decrease sink 
temperature as to increase source temperature. Therefore, for efficient operation of real gas power plant, high heat source inlet temperature should be achieved.

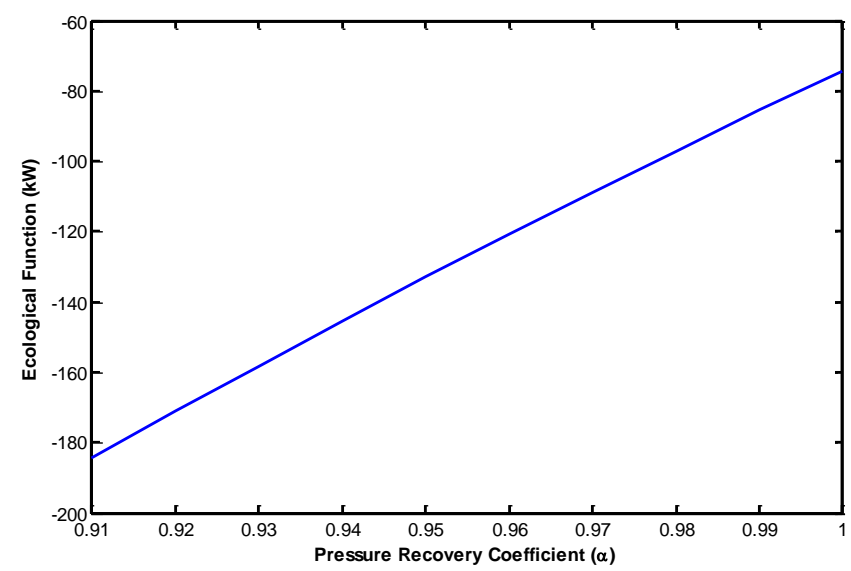

Fig. 5 (a) Variations of the Ecological Function with respect to pressure recovery coefficients

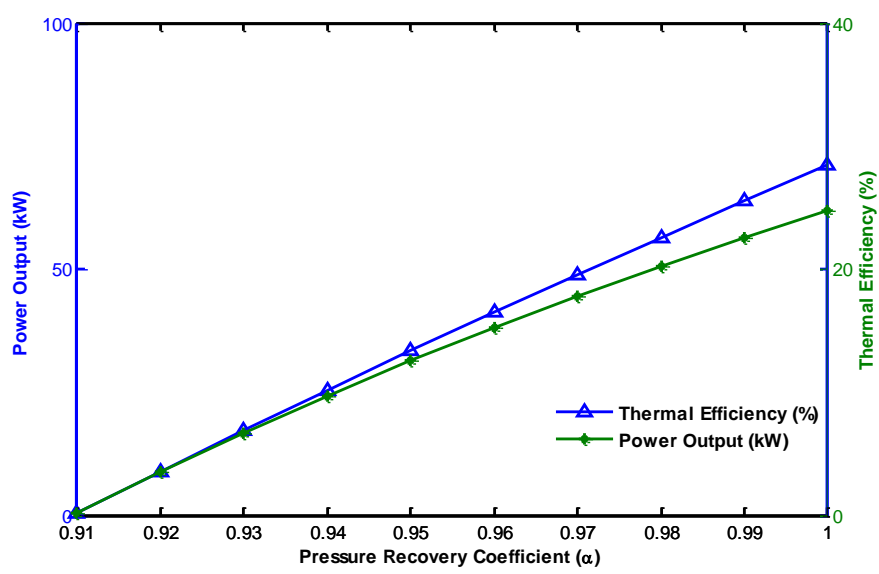

Fig. 5 (b) Variations of Power Output and Thermal Efficiency with respect to pressure recovery coefficients

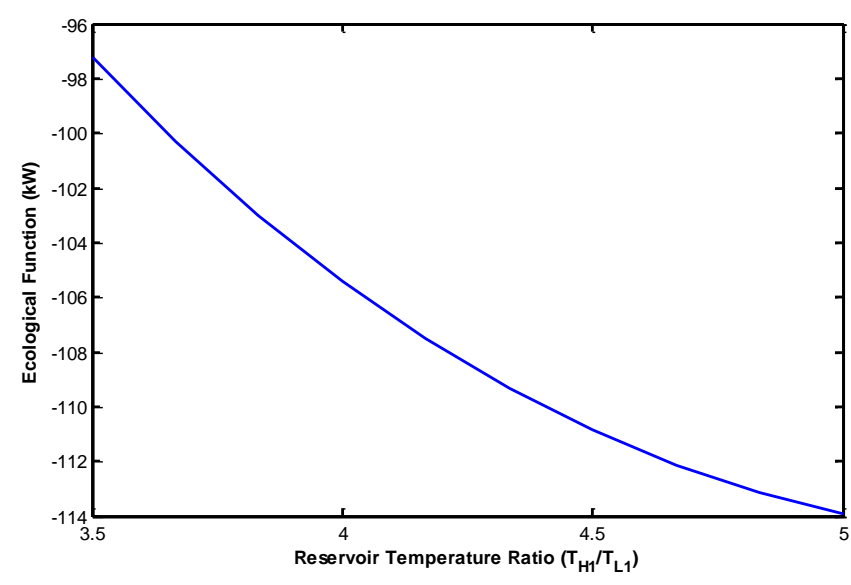

Fig 6(a) Variations of Ecological Function with respect to reservoir temperature ratio $\left(T_{\mathrm{H} 1} / T_{\mathrm{L} 1}\right)$

\section{Conclusion}

A more practical regenerative Brayton heat engine cycle model is examined in this paper. The ecological function is optimized with respect to cycle temperature and corresponding power output/thermal efficiency is calculated for a typical set of operating conditions. The ecological function, power output and thermal efficiency is increasing with effectiveness of either side heat exchanger, component efficiencies, heat capacitance rates of source and sink side, pressure recovery coefficients while its value is decreasing for heat capacitance rate of working fluid, $\mathrm{C}_{\mathrm{W}}$. It is also seen that the effect of turbine efficiency is more as compared with compressor efficiency on maximum economic function and the corresponding power output/ thermal efficiency. The interpolating results forms descending criterion of effectiveness of the model as $\varepsilon_{\mathrm{L}}, \varepsilon_{\mathrm{H}}$ and heat capacitance rates as $\mathrm{C}_{\mathrm{L}}, \mathrm{C}_{\mathrm{H}}$, and $\mathrm{C}_{\mathrm{W}}$. The above relationships are to be followed for better execution of real gas power plants. Hence, the present cycle model will be the benchmark to design and study a real cycle from thermodynamic view point

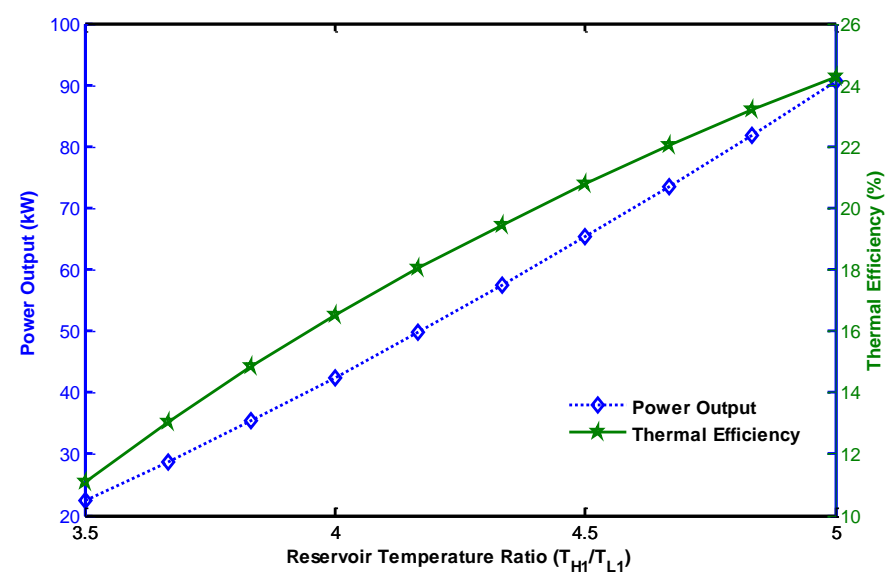

Fig 6(b) Variations of Power Output and Thermal Efficiency with respect to reservoir temperature ratio

\section{Nomenclature}

$\mathrm{A}=\operatorname{Area}\left(\mathrm{m}^{2}\right)$

$\mathrm{C}=$ Heat Capacitance Rate $\left(\mathrm{kWK}^{-1}\right)$

$\mathrm{k}=$ specific heat ratio

$\mathrm{N}=$ Number of heat transfer units

$\mathrm{P}=$ Power output $(\mathrm{kW})$

$\mathrm{Q}=$ Heat transfer rate $(\mathrm{kW})$

$\mathrm{T}=$ Temperature $(\mathrm{K})$

$\mathrm{U}=$ Overall heat transfer Coefficient $\left(\mathrm{kWm}^{-2} \mathrm{~K}^{-1}\right)$

\section{Greek letters:}

$\eta=$ Thermal efficiency

$\varepsilon=$ Effectiveness

\section{Subscripts:}

$\mathrm{H}=$ source side

$\mathrm{L}=$ sink side

$\mathrm{R}=$ regenerator side

$\mathrm{s}=$ ideal $/$ isentropic

$\mathrm{t}=$ turbine

$\mathrm{c}=$ compressor

$\mathrm{W}=$ working fluid

The different parameters are given as below:

$X=x_{1} x_{3}-x_{2} x_{4}$ 


$$
\begin{aligned}
& X_{1}=x_{12}\left(x_{5}^{2}-4 X y_{5}\right) \\
& x_{1}=\varepsilon_{R}(1-b)\left(1-\eta_{c}\right)+\eta_{c} \\
& x_{2}=(1-a)\left(1-\varepsilon_{R}\right) \\
& x_{3}=\alpha(1-a)\left(1-\eta_{t}^{-1}\right)\left(1-\varepsilon_{R}\right) \\
& x_{4}=(1-b) \varepsilon_{R} \\
& x_{5}=x_{1} y_{3}+x_{3} y_{1}-x_{4} y_{2}-x_{2} y_{4} \\
& x_{6}=x_{1} z_{3}+x_{3} z_{1}-x_{4} z_{2}-x_{2} z_{4} \\
& x_{7}=C_{W}\left\{a\left(1-\varepsilon_{R}\right)+b \varepsilon_{R}\right\} \\
& x_{8}=C_{W} a\left(1-\varepsilon_{R}\right) \\
& x_{9}=C_{W} T_{0}\left(a\left(1-\varepsilon_{R}\right) / T_{H 1}+b \varepsilon_{R} / T_{L 1}\right) \\
& x_{10}=x_{7}+x_{9} \\
& x_{11}=X y_{10}^{2} / x_{10}^{2}-x_{5} y_{10} / x_{10} \\
& x_{12}=x_{11}+y_{5} \\
& Y=x_{5} T_{4}+x_{6} \\
& Y_{1}=2 x_{12}\left(x_{5} x_{6}-2 X y_{6}\right) \\
& y_{1}=\left(1-\varepsilon_{R}\right)(1-b)\left(1-\eta_{c}\right) \\
& y_{2}=(1-a) \varepsilon_{R} \\
& y_{3}=\alpha\left\{\eta_{t}^{-1}+(1-a)\left(1-\eta_{t}^{-1}\right) \varepsilon_{R}\right\} \\
& y_{4}=\left(1-\varepsilon_{R}\right)(1-b) \\
& y_{5}=y_{1} y_{3}-y_{2} y_{4} \\
& y_{6}=y_{1} z_{3}+z_{1} y_{3}-z_{2} y_{4}-y_{2} z_{4} \\
& y_{7}=C_{W}\left\{b\left(1-\varepsilon_{R}\right)+a \varepsilon_{R}\right\} \\
& y_{8}=a C_{W} \varepsilon_{R} \\
& y_{9}=C_{W} T_{0}\left(a \varepsilon_{R} / T_{H 1}+b\left(1-\varepsilon_{R}\right) / T_{L 1}\right) \\
& y_{10}=y_{7}+y_{9} \\
& Z=y_{5} T_{4}^{2}+y_{6} T_{4}+z_{5} \\
& Z_{1}=x_{11}\left(x_{6}^{2}-4 X z_{5}\right)+y_{6}\left(x_{5} x_{6}-X y_{6}\right)-x_{5}^{2} z_{5} \\
& z_{1}=\left(1-\eta_{c}\right) b T_{L 1} \\
& z_{2}=a T_{H 1} \\
& z_{3}=\alpha a\left(1-\eta_{t}^{-1}\right) T_{H 1}
\end{aligned}
$$

$z_{5}=z_{1} z_{3}-z_{2} z_{4}$$$
z_{6}=C_{W}\left(a T_{H 1}+b T_{L 1}\right)
$$$$
z_{7}=C_{W} a T_{H 1}
$$$$
z_{8}=C_{W} T_{0}(a+b)
$$$$
z_{9}=z_{6}+z_{8}
$$

\section{References}

[1] Curzon F.L., Ahlborn B. Efficiency of Carnot heat engine at maximum power output. Am. J. Phys., 1975, 43: 22-24.

[2] Salamon P., Nitzan A. Finite time optimization of a Newton's law Carnot cycle. J. Chem. Phys., 1981, 74: 3446-3460.

[3] Leff H.S. Thermal efficiency at maximum power output: New results for old engine. Am. J. Phys., 1987, 55(7): 602-610.

[4] Wu C., Kiang R.L. Work and Power optimization of a finite time Brayton cycle. International Journal of Ambient Energy, 1990, 11(3): 129-136.

[5] Wu C. Power optimization of an endoreversible Brayton gas heat engine. Energy Convers. Mgmt.,

1991, 31 (6): 561-565.

[6] Wu C., Kiang R.L. Power performance of a nonisentropic Brayton cycle. Journal of Engineering for Gas Turbines and Power, 1991, 113: 501-504.

[7] Ibrahim O.M., Klein S. A., Mitchell J.W. Optimum heat power cycles for specified boundary conditions. $J$. Eng. Gas Turbine Power, 1991, 113 (4): 514-521.

[8] Angulo-Brown, F. An ecological optimization criterion for finite time heat engines. Journal of Applied Physics, 1991, 69(11): 7465-7469.

[9] Yan Z. Comment on ecological optimization criterion for finite time heat engines. Journal of Applied Physics, 1993, 73 (7): 3583.

[10] Cheng C.Y., Chen C.K.,Ecological optimization of an endoreversible Brayton cycle. Energy Conversion and Management, 1998, 39 (1/2): 33-44.

[11] Cheng C.Y., Chen C.K. Ecological optimization of an irreversible Brayton cycle. J. Phys. D: Appl. Phys., 1999, 32: 350-357

[12] Kaushik S.C., Tyagi S.K. Finite Time Thermodynamic analysis of an irreversible regenerative closed cycle Brayton heat engine. Int. J. of Solar Energy, 2002, 22 (3-4): 141-151.

[13] Tyagi S.K, Kaushik S C, Salhotra R. Ecological optimization and performance study of irreversible Stirling and Ericsson heat engines, Journal of Physics D: Applied Physics, 2002, 35 (20): 2668-2675. 
[14] Kaushik S.C., Tyagi S. K., Singhal M. K. Parametric study of an irreversible regenerative Brayton cycle with isothermal heat addition. Energy Conversion and Managemen, 2003, 44: 2013-2025.

[15] Tyagi S.K., Kaushik S.C., Tiwari V. Ecological optimization and parametric study of an irreversible regenerative modified Brayton cycle with isothermal heat addition. Entropy, 2003, 5: 377-390.

[16] Chen L., Zhou J., Sun F., Wu C. Ecological optimization for generalized irreversible carnot engines. Applied Energy, 2004, 77(3): 327-338.

[17] Ust Y., Safa A., Sahin B. Ecological performance analysis of an endoreversible regenerative Brayton heat engine. Applied Energy, 2005, 80 (3): 247-260.

[18] Ust Y., Sahin B., Kodal A. Performance analysis of an irreversible Brayton heat engine based on ecological coefficient of performance criterion. International Journal of Thermal Sciences, 2006, 45 (1): 94-101.

[19] Ust Yasin, Sahin B., Kodal A., Akcay IH. Ecological coefficient of performance analysis and optimization of an irreversible regenerative Brayton heat engine. Applied Energy, 2006, 83: 558-572.
[20] Xia D., Chen L., Sun F., Wu C. Universal ecological performance for endoreversible heat engine cycles. International Journal of Ambient Energy, 2006, 27 (1): 15-20.

[21] Tyagi S.K., Kaushik S.C. Ecological optimization of an irreversible regenerative intercooled Brayton heat engine with direct heat loss. International Journal of Ambient Energy, 2005, 26 (2): 81-92.

[22] Tyagi S. K., Chen J, Kaushik S C. Optimal criterion based on the ecological function of an irreversible intercooled regenerative modified Brayton cycle. Int. J. Exergy, 2005, 2(1): 90-107.

[23] Li J., Chen L., Sun F. Ecological performance of an endoreversible Carnot heat engine with complex heat transfer law. International Journal of thermal energy, 2011, 30: 55-64.

[24] Emin A. Models for optimum thermo-ecological criterion of actual thermal cycles. Thermal Science, 2013, 17(3): 915-930.

[25] Wang J., Chen L., Ge Y., Sun F. Ecological performance analysis of an endoreversible modified Brayton cycle. International Journal of Sustainable Energy, 2014, 33 (3): 619-634. 